\title{
Integrated High-Throughput Small RNA and Transcriptome Sequencing Unveil The Shape- Dependent Toxicity of Nano-Alumina in Rat Astrocytes
}

\section{Yuanyuan Chen}

Chinese Center for Disease Control and Prevention National Institute of Environmental Health

\section{Li Dong}

Chinese Center for Disease Control and Prevention National Institute of Environmental Health

Fuchang Deng

Chinese Center for Disease Control and Prevention National Institute of Environmental Health

\section{Yaqiang Cao}

Chinese Center for Disease Control and Prevention National Institute of Environmental Health

\section{Yuanzheng Fu}

Chinese Center for Disease Control and Prevention National Institute of Environmental Health

\section{Mu Zhu}

Chinese Center for Disease Control and Prevention National Institute of Environmental Health

\section{Guangqiu Qin}

GuangXi University of Chinese Medicine

\section{Dayna Schultz}

Aristotle University of Thessaloniki

\section{Kamran Shekh}

University of Alberta

\section{Song Tang ( $\nabla$ tangsong@nieh.chinacdc.cn )}

Chinese Center for Disease Control and Prevention National Institute of Environmental Health https://orcid.org/0000-0003-3219-1422

\section{Research}

Keywords: Nanoparticles, Morphology, High throughput sequencing, MicroRNAs, Transcriptome, Pathway analysis

Posted Date: June 15th, 2021

DOI: https://doi.org/10.21203/rs.3.rs-607858/v1 
License: (c) (i) This work is licensed under a Creative Commons Attribution 4.0 International License. Read Full License

Version of Record: A version of this preprint was published at Environmental Sciences Europe on August 16th, 2021. See the published version at https://doi.org/10.1186/s12302-021-00540-9. 


\section{Abstract \\ Background}

The large-scale applications of alumina nanoparticles $\left(\mathrm{Al}_{2} \mathrm{O}_{3}\right.$-NPs), one of the most important NPs in the global market, is causing severe damages to the environment and human health. Our previous research has revealed a critical role of nanoparticle morphology (e.g., flake and rod) in determining the toxic potencies of $\mathrm{Al}_{2} \mathrm{O}_{3}$-NPs, where nanorods demonstrated a significantly stronger toxic response than that of nanoflakes. However, their underlying mechanisms have not been completely elucidated yet. In the present study, we evaluated and compared the potential toxicological mechanisms of two shapes of $\gamma^{-}$ $\mathrm{Al}_{2} \mathrm{O}_{3}$-NPs (flake versus rod) by measuring miRNAs and mRNAs profiles of astrocytes in rat cerebral cortex, ex vivo.

\section{Results}

Totals of 269 mRNAs and 122 miRNAs, 180 mRNAs and 116 miRNAs were differentially expressed after nanoflakes or nanorods exposure, respectively. Among them, 55 miRNAs (e.g., miR-760-5p, miR-326-3p, and miR-35) and 105 mRNAs (e.g., Kdm4d, Wdr62, and Rps6) showed the same trend between the two shapes. These miRNAs and mRNAs were mainly involved in apoptosis, inflammatory pathways (e.g., NFkappa B), carcinogenic pathways (e.g., MAPK, p53, Notch, Rap1, and Ras), and cellular lipid metabolisms (e.g., glycerolipid metabolism, sphingolipid, and ether lipid metabolism). However, the remaining miRNAs and mRNAs either showed an opposite trend or only changed by a particular shape. Nanorods could specifically alter the changes of PI3K/Akt, AMPK and TNF pathways, cell cycle, and cellular senescence, while nanoflakes caused the changes of Toll and Imd signaling pathways.

\section{Conclusions}

In this study, we demonstrate that the toxicity of nanorods might be stronger than that of nanoflakes. And this study also demonstrates the critical role of morphology in nanotoxicity of nano-alumina and reveals its potential biomolecular mechanisms.

\section{Introduction}

With the rapidly increasing applications of nanoparticles (NPs), the annual output of NPs has sharply grown from 2300 tons in 2008 to nearly 58,000 tons in 2020 (Lewinski et al., 2008). Alumina nanoparticles ( $\mathrm{Al}_{2} \mathrm{O}_{3}$-NPs), one of the most important commercial NP productions in the global market, is widely used in pharmaceutical products, biomaterials, and high-energy systems (Sadiq et al., 2009; Keller et al., 2013). Unfortunately, the tremendous development of nanotechnology and excessive application of $\mathrm{Al}_{2} \mathrm{O}_{3}$-NPs has inevitably brought its increased burden to the environment and human health ( $\mathrm{Li}$ et al., 
2016). Although earlier epidemiological and toxicological studies have demonstrated that $\mathrm{Al}_{2} \mathrm{O}_{3}-\mathrm{NPs}$ exposure could lead to Alzheimer's disease and other neurodegenerative disorders (Townsend et al., 1985; Yokel, 2000), which could be linked to oxidative stress, inflammation, genotoxicity, and cell death (Balasubramanyam et al., 2009; Srikanth et al., 2015; Jalili et al., 2020; Li et al., 2020), their underlying toxicological mechanisms still remain to be understood.

Many factors significantly affect the toxicity of NPs, including their physical and chemical properties (e.g., size, shape, and structure characteristics), concentrations, incubation time, and receptor cell types (Jalili et al., 2020). Previous reports have mostly focused on $\mathrm{Al}_{2} \mathrm{O}_{3}-\mathrm{NPs}$ toxicities induced by particles of various sizes or structures (Park et al., 2015; Park et al., 2016); however, the toxicity induced by different particle shapes have been rarely reported. For instance, it was demonstrated that four different shapes of hydroxyapatite NPs could increase ROS production, alkaline phosphatase activity and the concentration of calcium, and decrease the mitochondrial membrane potential and lysosome integrity, the toxicity potential of four shapes were ranked as follows: plate $>$ sphere $>$ needle $>$ rod (Huang et al., 2019). In our previous study, the toxicities of $\mathrm{Y}-\mathrm{Al}_{2} \mathrm{O}_{3}$-NPs of two different shapes (flake versus rod) and their effects on metabolic profiles were compared in the astrocytes of rat cerebral cortex, ex vivo (Dong et al., 2019). Significantly stronger cytotoxicity and apoptosis were observed for nanorods than for nano-flakes, which were associated with significantly greater ROS accumulation, apoptosis, inflammation induction, and metabolic responses. However, the underlying mechanisms for the shape-induced nanotoxicity, especially at the microRNA (miRNA) and transcriptional (mRNA) expression levels, were not completely understood in the study.

Omics (e.g., epigenomics and transcriptomics) have been recognized as a comprehensive and efficient approach to identify new biomarkers and to elucidate specific mechanisms driving toxicological effects induced by nano-alumina (Fröhlich, 2017). For example, mRNA profiling has been used in $\mathrm{Al}_{2} \mathrm{O}_{3}$-NPexposed human bronchial epithelial (HBE) cells, showing differentially expressed genes encoding proteins essential for mitochondrial function, such as oxidative phosphorylation (Li et al., 2016). The toxicities of various surface modified $\mathrm{Al}_{2} \mathrm{O}_{3}-\mathrm{NPs}$ (pristine, hydrophile or lipophile) to Caenorhabditis elegans ( $C$. elegans) have been found to differ, with lipophilic NPs being the most toxic modification (Zhang et al., 2021). The lipophilic $\mathrm{Al}_{2} \mathrm{O}_{3}$-NPs could significantly induce excessive ROS production, destroy the redox balance, and lead to apoptosis (Yun et al., 2020). Moreover, transcriptomic analysis further showed that miR-297 was significantly up-regulated in HBE cells, inducing pulmonary inflammation via the Notch pathway (Yun et al., 2020). Furthermore, $\mathrm{Al}_{2} \mathrm{O}_{3}-\mathrm{NPs}$ could cause an increase in expressions of miR-395, miR-397, miR-398, and miR-399 in tobacco seedlings (Caitlin E. Burklew et al., 2012). However, to date, the integrated application of a variety of genetically related omics approaches to systematically evaluate the impact of different shapes of $\mathrm{Al}_{2} \mathrm{O}_{3}$-NPs exposure is relatively scarce.

As an extension of our previous work (Dong et al., 2019), in the present study, we exposed rat primary astrocytes to $\mathrm{Y}-\mathrm{Al}_{2} \mathrm{O}_{3}$-NPs of two distinct shapes (flake versus rod) at a dose of $125 \mu \mathrm{g} / \mathrm{mL}$ for $72 \mathrm{~h}$ with the aims to: (1) comprehensively characterize the differentially expressed miRNAs and mRNAs through 
high-throughput small RNA and transcriptome sequencings; (2) identify the miRNAs and mRNAs specifically responding to nanoflakes or nanorods; (3) explore the interactions between miRNA and mRNA responding to the distinct toxicities of nanoflakes and nanorods, for a further understanding of their underlying mechanisms or pathways.

\section{Materials And Methods}

\section{Nanoparticles preparation and sample collection}

$\mathrm{Y}-\mathrm{Al}_{2} \mathrm{O}_{3}$-NPs were successfully synthesized and fully characterized (e.g., particle size and shape) as reported in our previous study (Dong et al., 2019). Rat primary astrocytes were extracted from the cortex of neonatal $24 \mathrm{~h}$ old Wistar rats (purchased from the Chinese Experimental Animal Center of Military Medical Science Academy, Beijing, China) as previously described (Dong et al., 2019). Before the

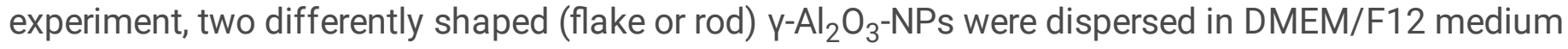
(HyClone, Beijing, China), sonicated and shaken for $30 \mathrm{mins}$ at $20^{\circ} \mathrm{C}$. Rat astrocytes were then exposed to nanoflakes and nanorods at the concentration of $125 \mu \mathrm{g} / \mathrm{mL}$ for $72 \mathrm{~h}$ with significant cytotoxicity

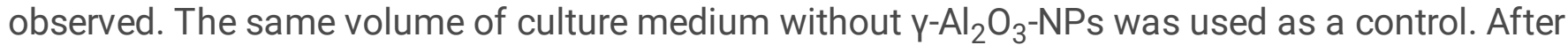
exposure, cells were washed in cold phosphate buffered saline (PBS) for three times and total RNA was isolated using TRIzol reagent (TaKaRa Bio, Osaka, Japan; Reuther et al., 2019).

\section{Total RNA extraction}

After exposure, total RNA was extracted from the cells using Trizol (Invitrogen, Carlsbad, CA, USA) according to the supplier's instructions. Briefly, samples were centrifuged for 5 mins with $12,000 \times \mathrm{g}$ at $4^{\circ} \mathrm{C}$, and supernatants were transferred into a new RNAase-free eppendorf tube with $0.3 \mathrm{~mL}$ chloroform/isoamyl alcohol (24:1). The mix was shaken for 15 seconds, and then centrifuged at $12,000 \times \mathrm{g}$ for 10 minutes at $4^{\circ} \mathrm{C}$. After centrifugation, RNA containing the upper aqueous phase was transferred into a new RNAase-free tube with equal volume of supernatant of isopropyl alcohol, then centrifuged at 12,000xrpm for 20 mins at $4^{\circ} \mathrm{C}$. After discarding the supernatant, RNA pellets were washed twice with $1 \mathrm{~mL}$ of $75 \%$ ethanol. The mix was then centrifuged at $12,000 \times \mathrm{rpm}$ for 3 mins at $4^{\circ} \mathrm{C}$ to collect residual ethanol, and then the pellet was allowed to air dry for 10 mins. Finally, $25 \mu \mathrm{L}$ of diethylpyrocarbonate (DEPC)-treated water was added to dissolve the RNA. Total RNA was qualified and quantified using a NanoDrop ND-2000 and Agilent 2100 bioanalyzer (Thermo Fisher Scientific, MA, USA).

\section{miRNA library preparation and sequencing}

A library of miRNA was prepared with $1 \mu \mathrm{g}$ total RNA for each sample. Total RNA was purified by electrophoretic separation on a $15 \%$ urea denaturing polyacrylamide gel electrophoresis (PAGE) gel. Small RNA regions with the 18-30 nt bands in the marker lane were excised and recovered. The 18-30 nt small RNAs were ligated to a 5'-adaptor and a 3'-adaptor. The adapter-ligated small RNAs were subsequently transcribed into cDNA by SuperScript II Reverse Transcriptase (Invitrogen, Waltham, MA, USA). PCR products were selected by agarose gel electrophoresis with target fragments 100-120 bp, and 
then purified by the QIAquick Gel Extraction Kit (QIAGEN, Valencia, CA, USA). The library was assessed for quality and quantity by checking the distribution of the fragment size using the Agilent 2100 bioanalyzer and by quantifying the library using qRT-PCR. The final ligation PCR products were sequenced using the BGISEQ-500 platform (BGI-Shenzhen, China).

\section{miRNA sequencing data processing}

The raw tags were processed using the following steps by removing low quality tags, tags with 5 primer contaminants or poly A, tags without 3 primer or insertion, and tags shorter than $18 \mathrm{nt}$. After filtering, the clean tags were mapped to the reference genomes and other sRNA databases, including miRbase, siRNA, piRNA, and snoRNA with Bowtie2. MiRanda, RNAhybrid, and TargetScan were used to predict the target genes of miRNAs. The expression level of miRNA was calculated by counting the absolute number of molecules using unique molecular identifiers. Differential expression analysis was performed using the DEGseq. $Q$ value $\leq 0.001$ and absolute value of Log2Ratio $\geq 1$ were the default threshold to indicate the significance. To annotate gene functions, all target genes were aligned against the Kyoto Encyclopedia of Genes (KEGG) and GO database. GO enrichment analysis (http://www.geneontology.org/) and KEGG enrichment analysis (https://www.kegg.jp/) of the target genes were performed using phyper in $\mathrm{R}$ software v3.3.1. The $p$ value was corrected using the Bonferroni method. GO terms or KEGG terms were defined as significantly enriched terms only when corrected $p$ value $\leq 0.05$.

\section{mRNA library preparation and sequencing}

Oligo(dT)-attached magnetic beads were used to purify mRNA. Purified mRNA was fragmented into small pieces with fragmentation buffer. First-strand CDNA was then generated using random hexamer-primed reverse transcription and followed by a second-strand cDNA synthesis. A-tailing mix and RNA index adapters were added by incubating to end repair. The cDNA fragments were amplified by PCR, and the products were purified by Ampure XP Beads. After dissolution in EB solution, the product was validated on the Agilent Technologies 2100 bioanalyzer. PCR products were heated, denatured, and circularized by the splint oligo sequence to obtain the final library. The single strand circle DNA was formatted as the final library, which was then amplified with phi29 to make DNA nanoballs (DNBs) > 300 copies per molecule. DNBs were loaded into the patterned nanoarray, and single end 50 base reads were generated on BGISEQ-500 platform (BGI-Shenzhen, China).

\section{mRNA sequencing data processing}

mRNA sequencing data was filtered with SOAPnuke (v1.5.2) by removing reads, which containing sequencing adapter, low-quality base ratio (base quality $<5$ ) $>20 \%$, and unknown base (' $N$ ' base) ratio $>$ $5 \%$ (Chen et al., 2018). Using HISAT2 (v2.0.4), the clean reads in FASTQ format were mapped to the reference genome. Then, Bowtie2 (v2.2.5) was applied to align the clean reads to the reference coding gene set, and then the expression level of gene was calculated by RSEM (v1.2.12). Differential expression analysis was performed using the DESeq2 (v1.4.5) with Q value $\leq 0.05$. GO and KEGG enrichment analyses of the annotated differentially expressed genes were performed by phyper based on 
Hypergeometric test. The significant levels of terms and pathways were corrected by $Q$ value with a rigorous threshold $(Q$ value $\leq 0.05)$ by Bonferroni method.

\section{Statistical analysis}

Statistical analysis was completed by using R software v3.3.1. Statistical analyses of the expression levels of miRNAs and mRNAs were evaluated by multiple comparisons of Kruskal- Wallis rank sum test using agricolae package. After normalization, the expression levels of miRNAs and mRNAs were imported into R for principal components analysis (PCA) by using packages of rop/s and ggplot2. Venn diagram and volcano plots were conducted by using the packages of VennDiagram and ggplot2, respectively. For the illustration of KEGG enrichment pathway analysis, bubble plots used ggplot2, ggrepel and RColorBrewer packages while heatmaps used pheatmap, ggplot2 and dplyr packages. The interaction network analysis between the miRNAs and differentially expressed genes used Cytoscape software (Shannon et al., 2003). Q values (FDR) $<0.05$ were considered as statistically significance for all data analysis.

\section{Results}

\subsection{Analysis of small RNA and transcriptome sequencing}

According to our previous research (Dong et al., 2019), although $\mathrm{\gamma}-\mathrm{Al}_{2} \mathrm{O}_{3}-\mathrm{NPs}$ of both shapes (flake or rod) caused a significant increase in toxicity in a dose-dependent manner in rat ASTs exposed to 31.3, 62.5 and $125 \mu \mathrm{g} / \mathrm{mL}$ for $72 \mathrm{~h}$, the cytotoxic and apoptotic effects caused by nanorods were found to be significantly stronger than those of nanoflakes at the same dose. In order to better understand the potential mechanisms of different levels of toxicities, the highest sublethal concentration of $125 \mu \mathrm{g} / \mathrm{mL}$ of nanoflakes or nanorods were chosen in the present experiments.

The raw reads, clean reads, and other basic information of miRNAs and mRNAs, through small RNA sequencing and transcriptome analysis, are summarized in Table 1. For small RNA sequencing, 27.11, 26.13, and 26.49 million clean reads were acquired. miRDeep2 software further detected 659, 671, and 647 unique miRNAs in control, nanoflake, and nanorod groups, respectively. Among these, 578, 586, and 583 were known miRNAs, and 81, 85, and 64 were novel miRNAs in control, nanoflake, and nanorod groups, respectively (Table 1). For transcriptomes, after data filtering, a total of $21.74,21.75$, and 21.73 million clean reads were obtained in control, nanoflake, and nanorod groups, respectively. 
Table 1

Overview of small RNA (miRNA) and transcriptome (mRNA) sequencing in rat ASTs exposed to two different shapes (flake or

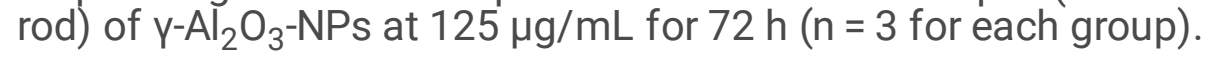

\begin{tabular}{|lllll|}
\hline Category & Data Type & Control & Flake & Rod \\
\hline miRNA & Total Raw Reads (M) & 29.32 & 28.59 & 28.95 \\
\cline { 2 - 5 } & Total Clean Reads (M) & 27.11 & 26.13 & 26.49 \\
\cline { 2 - 5 } & Clean Reads Q20 (\%) & 99.10 & 98.97 & 98.87 \\
\cline { 2 - 5 } & Clean Reads Ratio (\%) & 92.44 & 91.40 & 91.53 \\
\cline { 2 - 5 } & Total-miRNAs & 659 & 671 & 647 \\
\cline { 2 - 5 } mRNA & Known-miRNAs & 578 & 586 & 583 \\
\cline { 2 - 5 } & Novel-miRNAs & 81 & 85 & 64 \\
\cline { 2 - 5 } & Total Raw Reads (M) & 21.80 & 21.78 & 21.77 \\
\cline { 2 - 5 } & Total Clean Reads (M) & 21.74 & 21.75 & 21.73 \\
\cline { 2 - 5 } & Total Clean Bases (Gb) & 1.09 & 1.09 & 1.09 \\
\cline { 2 - 5 } & Clean Reads Q30 (\%) & 90.61 & 91.15 & 91.23 \\
\cline { 2 - 5 } & Clean Reads Ratio (\%) & 99.73 & 99.87 & 99.78 \\
\cline { 2 - 5 } & & & & \\
\cline { 2 - 4 } & & & & \\
\end{tabular}

Similarities and differences of the miRNAs and mRNAs profiles among three different groups were further assessed with principal components analysis (PCA) (Figs. 1A and 1B). Although both nanoflake and nanorod exposures drove separation from controls, largely along the PC 2 axis, the degree of separation of these two shapes was quite different, indicating that their toxicity mechanisms might be different.

\subsection{Significantly changed miRNAs and mRNAs with nanoflake and nanorod exposures}

Hierarchical cluster analyses of differentially expressed miRNAs and mRNAs in control, nanoflake, and nanorod groups is shown in Figure S1. Among Venn diagrams of control vs nanoflakes, control vs nanorods, and nanoflakes $v s$ nanorods, 15 miRNAs were significantly changed in both nanoflakes and nanorods, while 36 and 18 miRNAs were only found in nanoflake and nanorod exposures, respectively (Fig. 1C). Compared with control, volcano plots showed that 83 and 39 miRNAs were significantly upregulated and down-regulated, respectively, in nanoflakes, while 44 and 72 miRNAs were significantly upregulated and down-regulated, respectively, in nanorods (Figs. 2A and 2B). Therefore, some differences in miRNAs profiling were indeed found between nanoflakes and nanorods. Compared with the nanoflakes, there were 17 (12.59\%) and 83 (61.48\%) miRNAs significantly up-regulated and down-regulated in nanorods, respectively (Fig. 2C). 26 miRNAs were simultaneously increased in both nanoflake (23.21\%) and nanorod (22.41\%) groups, such as miR-151, miR-16, miR-24, miR-180, and miR-121, while 29 miRNAs were simultaneously decreased in both nanoflake (23.77\%) and nanorod (25\%) groups, such as miR-155, 
miR-35, miR-54, miR-65, and miR-164. Compared with the nanoflakes, 18 (15.52\%) miRNAs (e.g., miR-46, miR-89, miR-178, miR-150, and miR-49) were specifically increased in the nanorod group, while totally 43 (37.07\%) miRNAs (e.g., miR-55, miR-105, miR-109, miR-11, and miR-120) were specifically decreased. 57 (46.72\%) miRNAs (e.g., miR-70, miR-127, miR-61, miR-62, and miR-98) were specifically increased in the nanoflake group, while totally 10 (8.20\%) miRNAs (e.g., miR-60, miR-113, miR-169, and miR-40) were specifically decreased.

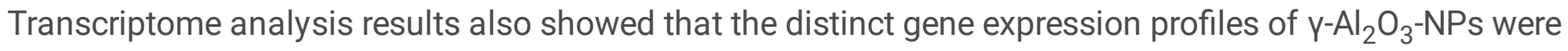
induced by the two different shapes. Compared with control, 222 mRNAs were significantly changed in both exposure groups, while 204 and 397 mRNAs were uniquely found in nanoflakes and nanorods groups, respectively (Fig. 1D). Similarly, volcano plots showed that 194 and 75 mRNAs were significantly up-regulated and down-regulated, respectively, in nanoflakes, while 130 and 50 mRNAs were significantly up-regulated and down-regulated, respectively, in nanorods (Figs. 2E and 2F). Compared with the nanoflakes, there were 77 (42.78\%) and 53 (29.44\%) mRNAs significantly up-regulated and downregulated in nanorods, respectively (Fig. 2G). 89 mRNAs were simultaneously increased in both nanoflakes (33.09\%) and nanorods (49.44\%), such as Kdm4d, Wdr62, and Rps6, while 16 mRNAs were simultaneously decreased in nanoflakes (5.95\%) and nanorods (8.89\%), such as LOC103692976, LOC103690175, and LOC100912475. Compared with nanoflakes, 41 (22.78\%) mRNAs (e.g., Dmrtc1a, Pou4f1, and Crkl) were specifically increased in nanorods, while 34 (18.89\%) mRNAs (e.g., Fam98b, Pkhd1, and Rp/37) were specifically decreased in total. 105 (39.03\%) mRNAs (e.g., Adora3, Sema3g, and Mmp28) were specifically increased in nanoflakes, while 59 (21.93\%) mRNAs (e.g., Cftr, Akr1b8, and $\mathrm{Cc} 2 \mathrm{~d} 2 \mathrm{~b}$ ) were specifically decreased. In general, both shapes of $\mathrm{Y}-\mathrm{Al}_{2} \mathrm{O}_{3}-\mathrm{NPs}$ significantly changed the profiling of miRNAs and mRNAs in rat ASTs, and the two shapes have different toxicity-related gene expression profiles.

\subsection{Pathway enrichment analysis of nanoflakes and nanorods modulated miRNAs and mRNAs}

KEGG pathway analysis revealed the linkages between our enriched data and cell signaling pathways (Kanehisa et al., 2019). In order to explore the possible mechanisms of shape dependent nanotoxicity, we performed the KEGG pathway enrichment analysis of the differentially expressed miRNAs and mRNAs in rat ASTs after nanoflake and nanorod exposures. Nanorods had a broader impact on the miRNA-induced pathways than that of nanoflakes. Compared with control, significantly changed miRNAs affected 97 signaling pathways after nanoflake exposure, while 141 signaling pathways were changed after nanorod exposure. There are 79 identical pathways both in nanoflakes (81.44\%) and nanorods (56.03\%) (e.g., NFkappa B, MAPK, and Rap1), and 18 (18.56\%) and 62 (43.97\%) specific pathways were observed in nanoflakes (e.g., Toll, pyruvate metabolism, and butanoate metabolism) and nanorods (e.g., phosphatidylinositol 3-kinase (PI3K)/Akt, AMPK, TNF), respectively (Figs. 3A, 3B, 3C and S2).

Similar to miRNA pathway enrichment findings, nanorods had a greater impact on the mRNA-induced pathways than that of nanoflakes. 13 and 23 signaling pathways were significantly changed by nanoflake and nanorod exposures, respectively. There are 6 identical pathways both in nanoflakes 
(46.16\%) and nanorods (26.09\%; e.g., Notch, sphingolipid metabolism, and ether lipid metabolism), 7 specific pathways in nanoflakes (53.84\%; e.g., Toll, ABC transporters, and folate biosynthesis), and 17 specific pathways in nanorods (74.91\%; e.g., ErbB, AMPK, cancer related pathways). Further, we found that both nanoflake and nanorod exposures could cause changes in environmental information processing-related signaling pathways (e.g., mTOR, Wnt, NF-kappa B, Hippo, and calcium signaling pathways; Figs. 3D, 3E, and S3). Compared with nanoflakes, the levels of lipolysis, apoptosis, and HIF-1 signaling pathways were significantly higher in nanorods (Fig. 3F). Furthermore, nanorod exposure was associated with the occurrence and development of multiple cancers, such as endometrial cancer, renal cell carcinoma, and non-small cell lung cancer (Figs. 3B and 3D). Altogether, KEGG results revealed that both shapes could activate inflammation and oncogenic signaling pathways, as well as change lipid metabolism in rat ASTs. Furthermore, nanorods have a more significant impact on these pathways compared to nanoflakes.

\subsection{Interaction Network Of Mirnas And Mrna}

MiRNAs function post-transcriptionally by generally base-pairing with the 3'-untranslated regions of mRNAs to repress protein synthesis in animals, plants, and protozoa (Fabian et al., 2010). Therefore, we combined small RNA and transcriptome sequencing results to determine the transcriptional changes related to miRNA perturbations. Based on the interaction network of the significantly altered miRNAs and genes (mRNAs) constructed by Cytoscape, a unique interaction relationship between differentially expressed genes and miRNAs was observed between two different shapes (Fig. 4).

Compared with control, the expression of miRNAs (e.g., miR-760-5p, miR-383-3p, and miR-326-3p) were significantly up-regulated in nanoflakes. These miRNAs positively regulated the expressions of $P d e 2 a$, Dpys/4, Elfn2, Tspan2 and Col23a1 and negatively regulated the expressions of Itga10 and Gbp1 (Fig. 4A). Meanwhile, the expressions of known miRNAs (e.g., miR-504, miR-483-3p, and miR-383-3p) were significantly up-regulated in nanorods. A part of these miRNAs positively regulated the expressions of Elfn2, Gpr62 and Faim2, and some miRNAs negatively regulated the expression of Grm3 (Fig. 4B). Compared with nanorods, the expressions of miRNAs and genes, such as miR-55, miR-210-5p, No/8, and Prrg3, were significantly down-regulated in nanoflakes (Fig. 4C). In addition, the significantly up-regulated or down-regulated miRNAs (e.g., miR-20, miR-74, and miR-127) were regarded as potential nanotoxicityrelated genes.

\section{Discussion}

The adverse impact of $\mathrm{Al}_{2} \mathrm{O}_{3}$-NPs on environment and human health has increased recently due to its wide application globally. They have been proven to exhibit neurotoxicity, genotoxicity, cytotoxicity, and developmental toxicity in animal and human cellular models (Zhu et al., 2008; Li et al., 2013; Sliwinska et al., 2015; Liu et al., 2019), mainly through the alternations in biomolecular pathways relevant to the accumulation of ROS, mitochondrial damages, immune disorders, and tissue injuries (Park et al., 2015; 
Srikanth et al., 2015; Li et al., 2016; Park et al., 2016). As different shapes of NPs could interact and immobilize differently with plasma proteins, their corresponding toxicity profiles might be different in a shape-dependent manner. Although previous studies have demonstrated a size-dependent toxicity of $\gamma^{-}$ $\mathrm{Al}_{2} \mathrm{O}_{3}$-NPs (Ates et al., 2015), comparing toxicological studies on differently shaped NPs (e.g., spheroidal, rod-like particles) have shown inconsistent results. In our previous study, a significant shape-dependent nanotoxicity was found (Dong et al., 2019). Exposure to two different shapes of $\mathrm{Y}-\mathrm{Al}_{2} \mathrm{O}_{3}-\mathrm{NPs}$ (flake versus rod) could induce a significant cytotoxicity in rat ASTs in a dose-dependent manner, including significantly increased concentrations of inflammatory factors (IL-1 $\beta$, IL-2, and IL-6) and alternations in the metabolism of amino acids, lipids and purines, and pyrimidines. In the present study, to further clarify the role of morphology in distinct nanotoxicities, the significantly changed miRNAs and mRNAs were

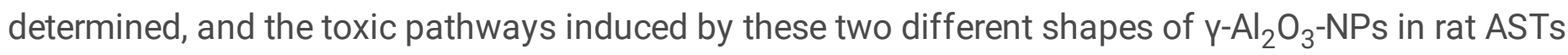
were further elucidated and compared through small RNA and transcriptomics sequencings.

Here we found that both shapes of $\mathrm{Y}-\mathrm{Al}_{2} \mathrm{O}_{3}$-NPs induced a few consistent changes in miRNAs (e.g., miR760-5p, miR-326-3p, and miR-35), mRNAs (e.g., Elfn2, Gpr62, and Kif26a), and signaling pathways (e.g., MAPK, Rap1 and $\mathrm{Ca}^{2+}$ signaling). More specifically, both shapes simultaneously induced the upregulations of miR-760-5p and miR-326-3p as well as the down-regulation of miR-35, suggesting their cytotoxic, neurotoxic, and carcinogenic potential. For example, the up-regulation of miR-760-5p has been linked to some neurotoxic symptoms (e.g., ataxic gait and head bobbing) in rats after 3,3'iminodipropionitrile exposure (Ogata et al., 2016). Knockdown of miR-35 could result in an enhancement of MAPK signal transduction and a significant increase in C. elegans germline death (Tran et al., 2019). Interaction network analysis between the significantly altered miRNAs and mRNAs showed that both shapes up-regulated miR-326-3p and its downstream target gene Elfn2, which was essential for mGluRs signaling properties and brain function. Elfn2 knockout mice showed a series of neuropsychiatric symptoms, including seizure susceptibility, hyperactivity disorder, and anxiety/compulsivity, suggesting that $\mathrm{y}-\mathrm{Al}_{2} \mathrm{O}_{3}-\mathrm{NPs}$ exposure might induce neurotoxicity through Elfn2 (Dunn et al., 2019). Further, KEGG pathway analysis showed that the significantly changed miRNAs and mRNAs by both shapes were mainly enriched in MAPK, Rap1, and calcium signaling pathways. Intracellular $\mathrm{Ca}^{2+}$ signaling is fundamental to neuronal physiology and viability, and the disruption in $\mathrm{Ca}^{2+}$ could lead to a variety of disease processes, especially neurodegenerative diseases (Demuro et al., 2010). MAPK signaling pathway plays an important role in cell growth, development, differentiation, and apoptosis and contributes to the occurrence of neurodegenerative disorders (Kim and Choi, 2015). In addition, Rap1 is mainly involved in the regulation of cell adhesion, which is over-activated in various tumors (e.g., breast cancers, prostate cancers, and melanoma), and plays a critical role in the migration, invasion, and metastasis of tumor cells (Hattori and Minato, 2003; Ichikawa et al., 2018). Lin et al. found that graphene oxide NP exposure could trigger neuronal differentiation through Rap1-TRPC2-ERK1/2 pathway (Lin et al., 2020). Altogether, these results show that there were certain consistent perturbations in miRNAs and mRNAs after two shapes of $\mathrm{Y}-\mathrm{Al}_{2} \mathrm{O}_{3}$-NPs exposure, indicating that these changes were mediated through $\mathrm{Y}-\mathrm{Al}_{2} \mathrm{O}_{3}-\mathrm{NPs}$, irrespective of its morphology. 
Moreover, morphology played an important role in the process of nanotoxicity. We previously observed that nanorods could induce a significantly higher apoptosis than that of nanoflakes, indicating its stronger toxicity (Dong et al., 2019). In agreement with previous study, some differences in miRNA and mRNA perturbations between the two shapes were observed in this study. Compared to nanoflakes, nanorods could significantly up-regulate miR-55 and miR-150, suggesting that nanorods could have a greater cytotoxicity and carcinogenicity potential. For instance, Alizadeh et al. found that inhibition of miR-55 could increase the activity of Caspase-3 and induce apoptosis in Jurkat cells (Alizadeh et al., 2014). The level of miR-150 in patients with nasopharyngeal carcinoma was significantly increased and correlated with tumor distant metastasis and recurrence, and shortened the overall survival of patients (Liu et al., 2018). Further, integration analysis showed that nanorods did not only cause the up-regulation of Elfn, but also up-regulate the expression of Tspan2 and Col23a1, which were both the downstream target genes of miR-326-3p. Tspan2 was positively correlated with the neuroinflammation and carcinogenesis, and Tspan 2 knockdown suppressed the metastasis to lung and liver, enabling prolonged survival (Otsubo et al., 2014). Furthermore, compared with nanoflakes, the changes in signaling pathways were significantly induced by nanorods. Nanorods could especially activate PI3K/Akt, AMPK, and TNF signaling pathways. For example, the levels of neuroinflammatory cytokines (TNF-alpha, IL-6, and IL-1 $\beta$ ) were increased and memory and learning abilities were impaired in $\mathrm{AlCl}_{3}$-induced $\mathrm{AD}$ rat models, which was consistent with the previous results that $\mathrm{Y}-\mathrm{Al}_{2} \mathrm{O}_{3}-\mathrm{NPs}$ could induce an inflammatory response (Yin et al., 2020). PI3K/Akt was closely related to cell apoptosis, the occurrence and development of tumors, and energy metabolism (Ogawara et al., 2002; Kane and Weiss, 2003). Yang et al. found that $\mathrm{PM}_{2.5}$ exposure could cause the myocardial ADRB2 hypermethylation and activate the PI3K/Akt pathway in AC16 cells, resulting in cardiomyocyte apoptosis as well as cardiac dysfunction (Yang et al., 2019). In addition, compared with nanorods, nanoflakes could greatly activate the Toll and Imd signaling pathways, suggesting that nanoflakes could activate innate immune pathways. These studies demonstrate that nanorods might have stronger neurotoxicity, carcinogenicity, metabolic toxicity, and apoptotic ability, which might be mediated via morphology.

\section{Conclusion}

This is the first ex vivo study providing comprehensive evidence of distinct expression patterns regarding the changes of miRNAs and mRNAs in rat ASTs after two different types of $\mathrm{Y}-\mathrm{Al}_{2} \mathrm{O}_{3}$-NP exposures (Fig. 5). Both shapes induced identical changes in miRNAs and mRNAs related to apoptosis, inflammatory pathways (e.g., NF-kappa B), carcinogenic pathways (e.g., MAPK, p53, Notch, Rap1, and Ras), and cellular lipid metabolism (e.g., glycerolipid metabolism, sphingolipid, and ether lipid metabolism). However, the two different shapes also produced some distinct responses. It is evidenced that nanorods could specifically lead to the changes of PI3K/Akt, AMPK and TNF pathways, cell cycle, and cellular senescence, while nanoflakes specifically cause the changes of Toll and Imd signaling pathways, indicating that the previously observed higher toxicity with nanorods than nanoflakes, might be mediated through these differentially activated inflammatory and carcinogenic pathways and apoptosis. Further in vivo studies are highly needed to verify these findings. The present study sheds light on the potential 
toxic mechanisms caused by the morphology of NPs, and demonstrates that multi-omics is a powerful approach to identify morphology-related toxicological signaling pathways, which could offer new intervention targets for the future treatment of NP-exposure related diseases.

\section{Declarations}

\section{Ethics approval and consent to participate}

All animal experiments performed during this study were approved by the Institutional Animal Care and Use Committee (IACUC) of the Chinese Center for Disease Control and Prevention (China CDC) No. 2016003. All the authors read and approved the final manuscript. The present findings have not been subjected to any peer and policy review from China $\mathrm{CDC}$, and therefore does not necessarily reflect the views of the China CDC and no official endorsement should be inferred.

\section{Consent for publication}

All authors approve this manuscript to be published in Environmental Sciences Europe.

\section{Availability of data and materials}

The authors guarantee the authenticity of data and materials.

\section{Competing interests}

The authors declare no conflicts of interest.

\section{Funding}

This study was financially supported by the grants from the National Natural Science Foundation of China No. 21707132, Young Scholar Scientific Research Foundation of China CDC No. 2018A201, and Start-up Funding from NIEH, China CDC to Prof. Tang.

\section{Author Contributions}

Yuanyuan Chen: data curation, validation, and paper writing. Li Dong: conceptualization, methodology, conducting experiments and paper writing. Fuchang Deng: investigation and data visualization. Yaqiang Cao: data compilation and visualization. Yuanzheng Fu: literature search and data visualization. Mu Zhu: statistical analysis and interpretation. Guangqiu Qin: conceptualization and methodology. Dayna Schultz: reviewing and editing. Kamran Shekh: reviewing and editing. Song Tang: funding acquisition, investigation, project administration, paper writing, and supervision.

\section{Acknowledgements}

Many thanks to Prof. Hongxing Dai and Xingtian Zhao from the Department of Chemistry and Chemical Engineering of Beijing University of Technology for their boundless helps in synthesizing and 
characterizing $\mathrm{Y}-\mathrm{Al}_{2} \mathrm{O}_{3}-\mathrm{NPs}$.

\section{References}

1. Alizadeh S, Kaviani S, Soleimani M, Abroun S, Kashani-Khatib Z, Asgharzadeh A, Dargahi H, Mousavi R (2014) Mir-55 inhibition can reduce cell proliferation and induce apoptosis in Jurkat (Acute T cell Leukemia) cell line. Iranian journal of pediatric hematology oncology 4:141-150

2. Ates M, Demir V, Arslan Z, Daniels J, Farah IO, Bogatu C (2015) Evaluation of alpha and gamma aluminum oxide nanoparticle accumulation, toxicity, and depuration in Artemia salina larvae. Environmental toxicology 30:109-118

3. Balasubramanyam A, Sailaja N, Mahboob M, Rahman MF, Misra S, Hussain SM, Grover P (2009) Evaluation of genotoxic effects of oral exposure to aluminum oxide nanomaterials in rat bone marrow. Mutat Res 676:41-47

4. Burklew CE, Ashlock J, Winfrey WB, Zhang B (2012) Effects of Aluminum Oxide Nanoparticles on the Growth, Development, and microRNA Expression of Tobacco. PLoS One 7:e34783

5. Chen Y, Chen Y, Shi C, Huang Z, Zhang Y, Li S, Li Y, Ye J, Yu C, Li Z, Zhang X, Wang J, Yang H, Fang L, Chen Q (2018) SOAPnuke: a MapReduce acceleration-supported software for integrated quality control and preprocessing of high-throughput sequencing data. GigaScience 7:1-6

6. Demuro A, Parker I, Stutzmann GE (2010) Calcium signaling and amyloid toxicity in Alzheimer disease. J Biol Chem 285:12463-12468

7. Dong L, Tang S, Deng F, Gong Y, Zhao K, Zhou J, Liang D, Fang J, Hecker M, Giesy JP, Bai X, Zhang H (2019) Shape-dependent toxicity of alumina nanoparticles in rat astrocytes. Sci Total Environ 690:158-166

8. Dunn HA, Zucca S, Dao M, Orlandi C, Martemyanov KA (2019) ELFN2 is a postsynaptic cell adhesion molecule with essential roles in controlling group III mGluRs in the brain and neuropsychiatric behavior. Molecular psychiatry 24:1902-1919

9. Fabian MR, Sonenberg N, Filipowicz W (2010) Regulation of mRNA translation and stability by microRNAs. Annual review of biochemistry 79:351-379

10. Fröhlich E (2017) Role of omics techniques in the toxicity testing of nanoparticles. Journal of nanobiotechnology 15:84

11. Hattori M, Minato N (2003) Rap1 GTPase: functions, regulation, and malignancy. J BioChem 134:479-484

12. Huang LH, Sun XY, Ouyang JM (2019) Shape-dependent toxicity and mineralization of hydroxyapatite nanoparticles in A7R5 aortic smooth muscle cells. Scientific reports 9:18979

13. Ichikawa H, Itsumi M, Kajioka S, Maki T, Lee K, Tomita M, Yamaoka S (2018) Overexpression of exchange protein directly activated by CAMP-1 (EPAC1) attenuates bladder cancer cell migration. Biochem Biophys Res Commun 495:64-70 
14. Jalili P, Huet S, Lanceleur R, Jarry G, Le Hegarat L, Nesslany F, Hogeveen K, Fessard V (2020) Genotoxicity of Aluminum and Aluminum Oxide Nanomaterials in Rats Following Oral Exposure. Nanomaterials 10

15. Kane LP, Weiss A (2003) The PI-3 kinase/Akt pathway and T cell activation: pleiotropic pathways downstream of PIP3. Immunological reviews 192:7-20

16. Kanehisa M, Sato Y, Furumichi M, Morishima K, Tanabe M (2019) New approach for understanding genome variations in KEGG. Nucleic acids research 47:D590-Dd595

17. Keller AA, McFerran S, Lazareva A, Suh S (2013) Global life cycle releases of engineered nanomaterials. Journal of Nanoparticle Research 15

18. Kim EK, Choi EJ (2015) Compromised MAPK signaling in human diseases: an update. Arch Toxicol 89:867-882

19. Lewinski N, Colvin V, Drezek R (2008) Cytotoxicity of nanoparticles. Small 4:26-49

20. Li H, Huang T, Wang Y, Pan B, Zhang L, Zhang Q, Niu Q (2020) Toxicity of alumina nanoparticles in the immune system of mice. Nanomedicine 15:927-946

21. Li X, Zhang C, Zhang X, Wang S, Meng Q, Wu S, Yang H, Xia Y, Chen R (2016) An acetyl-L-carnitine switch on mitochondrial dysfunction and rescue in the metabolomics study on aluminum oxide nanoparticles. Part Fibre Toxicol 13:4

22. Li Y, Yu S, Wu Q, Tang M, Wang D (2013) Transmissions of serotonin, dopamine, and glutamate are required for the formation of neurotoxicity from Al203-NPs in nematode Caenorhabditis elegans. Nanotoxicology 7:1004-1013

23. Lin L, Zhuang X, Huang R, Song S, Wang Z, Wang S, Cheng L, Zhu R (2020) Size-Dependent Effects of Suspended Graphene Oxide Nanoparticles on the Cellular Fate of Mouse Neural Stem Cells. Int J Nanomed 15:1421-1435

24. Liu B, Tan Z, Jiang Y, Chen Y, Chen Y, Ling K (2018) Correlation between the expression of miR150 and FOXO4 and the local recurrence and metastasis of nasopharyngeal carcinoma after intensive radiotherapy. Journal of BUON 23:1671-1678

25. Liu X, Tang J, Song B, Zhen M, Wang L, Giesy JP (2019) Exposure to Al(2)O(3) nanoparticles facilitates conjugative transfer of antibiotic resistance genes from Escherichia coli to Streptomyces. Nanotoxicology 13:1422-1436

26. Ogata K, Kushida M, Miyata K, Sumida K, Takeda S, Izawa T, Kuwamura M, Yamate J (2016) Alteration of microRNA expressions in the pons and medulla in rats after 3,3'-iminodipropionitrile administration. Journal of toxicologic pathology 29:229-236

27. Ogawara Y, Kishishita S, Obata T, Isazawa Y, Suzuki T, Tanaka K, Masuyama N, Gotoh Y (2002) Akt enhances Mdm2-mediated ubiquitination and degradation of p53. J Biol Chem 277:21843-21850

28. Otsubo C, Otomo R, Miyazaki M, Matsushima-Hibiya Y, Kohno T, Iwakawa R, Takeshita F, Okayama H, Ichikawa H, Saya H, Kiyono T, Ochiya T, Tashiro F, Nakagama H, Yokota J, Enari M (2014) TSPAN2 is involved in cell invasion and motility during lung cancer progression. Cell reports 7:527-538 
29. Park EJ, Lee GH, Shim JH, Cho MH, Lee BS, Kim YB, Kim JH, Kim Y, Kim DW (2015) Comparison of the toxicity of aluminum oxide nanorods with different aspect ratio. Arch Toxicol 89:1771-1782

30. Park EJ, Lee GH, Yoon C, Jeong U, Kim Y, Cho MH, Kim DW (2016) Biodistribution and toxicity of spherical aluminum oxide nanoparticles. Journal of applied toxicology 36:424-433

31. Reuther J, Roy A, Monzon FA (2019) Transcriptome Sequencing (RNA-Seq). Genomic Applications in Pathology

32. Sadiq IM, Chowdhury B, Chandrasekaran N, Mukherjee A (2009) Antimicrobial sensitivity of Escherichia coli to alumina nanoparticles. Nanomedicine 5:282-286

33. Shannon P, Markiel A, Ozier O, Baliga NS, Wang JT, Ramage D, Amin N, Schwikowski B, Ideker T (2003) Cytoscape: a software environment for integrated models of biomolecular interaction networks. Genome research 13:2498-2504

34. Sliwinska A, Kwiatkowski D, Czarny P, Milczarek J, Toma M, Korycinska A, Szemraj J, Sliwinski T (2015) Genotoxicity and cytotoxicity of ZnO and Al203 nanoparticles. Toxicol Mech Methods $25: 176-183$

35. Srikanth K, Mahajan A, Pereira E, Duarte AC, Rao JV (2015) Aluminium oxide nanoparticles induced morphological changes, cytotoxicity and oxidative stress in Chinook salmon (CHSE-214) cells. Journal of applied toxicology 35:1133-1140

36. Townsend MC, Enterline PE, Sussman NB, Bonney TB, Rippey LL (1985) Pulmonary function in relation to total dust exposure at a bauxite refinery and alumina-based chemical products plant. Am Rev Respir Dis 132:1174-1180

37. Tran AT, Chapman EM, Flamand MN, Yu B, Krempel SJ, Duchaine TF, Eroglu M, Derry WB (2019) MiR35 buffers apoptosis thresholds in the C. elegans germline by antagonizing both MAPK and core apoptosis pathways. Cell death differentiation 26:2637-2651

38. Yang X, Zhao T, Feng L, Shi Y, Jiang J, Liang S, Sun B, Xu Q, Duan J, Sun Z (2019) PM(2.5)-induced ADRB2 hypermethylation contributed to cardiac dysfunction through cardiomyocytes apoptosis via PI3K/Akt pathway. Environment international 127:601-614

39. Yin S, Ran Q, Yang J, Zhao Y, Li C (2020) Nootropic effect of neferine on aluminium chloride-induced Alzheimer's disease in experimental models. J Biochem Mol Toxicol 34:e22429

40. Yokel RA (2000) The toxicology of aluminum in the brain: a review. Neurotoxicology 21:813-828

41. Yun J, Yang H, Li X, Sun H, Xu J, Meng Q, Wu S, Zhang X, Yang X, Li B, Chen R (2020) Up-regulation of miR-297 mediates aluminum oxide nanoparticle-induced lung inflammation through activation of Notch pathway. Environmental pollution 259:113839

42. Zhang S, Chu Q, Zhang Z, Xu Y, Mao X, Zhang M (2021) Responses of Caenorhabditis elegans to various surface modifications of alumina nanoparticles. Environmental pollution 271:116335

43. Zhu X, Zhu L, Duan Z, Qi R, Li Y, Lang Y (2008) Comparative toxicity of several metal oxide nanoparticle aqueous suspensions to Zebrafish (Danio rerio) early developmental stage. Journal of environmental science health Toxic/hazardous substances environmental engineering 43:278-284 
Figures
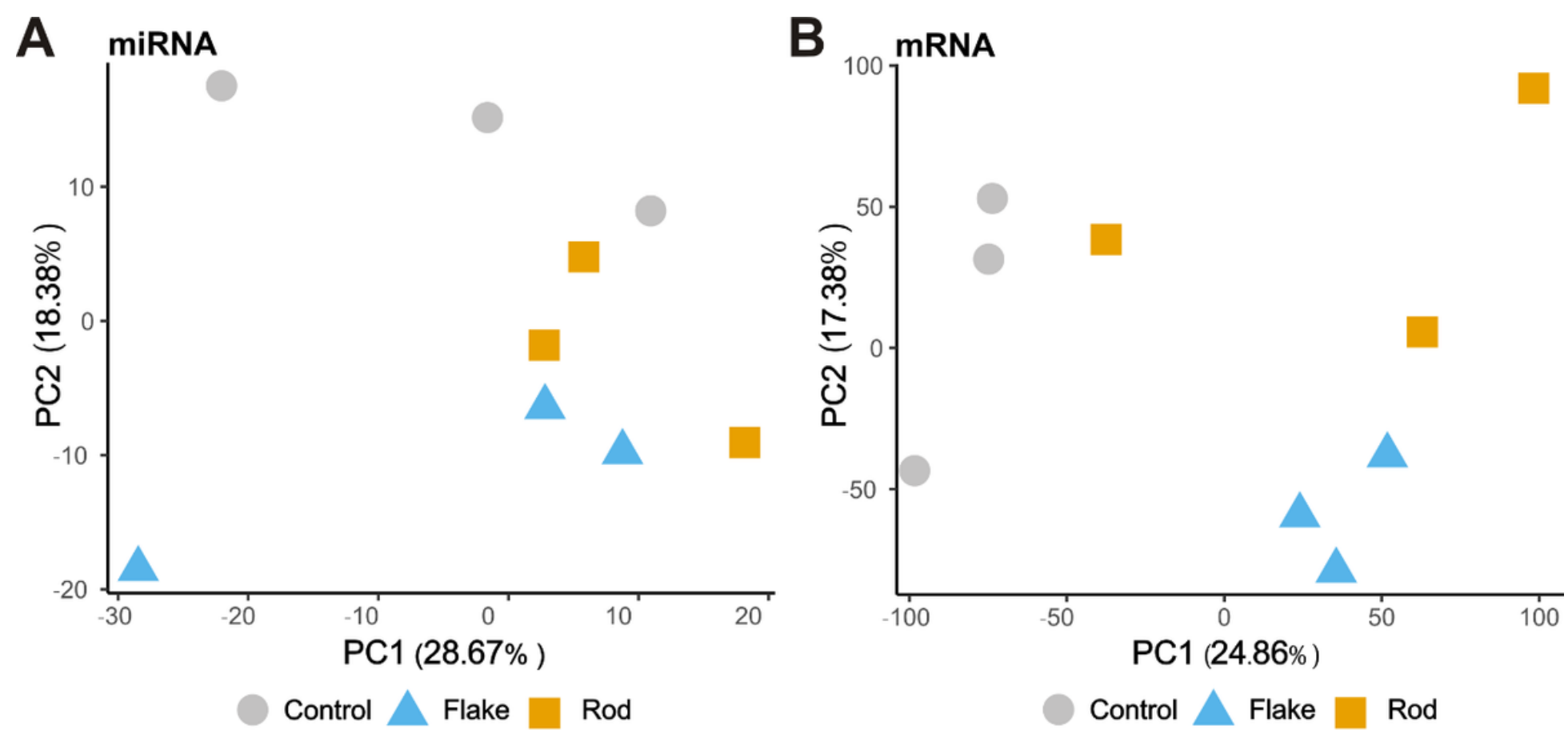

C

Control

Flake

Rod

D
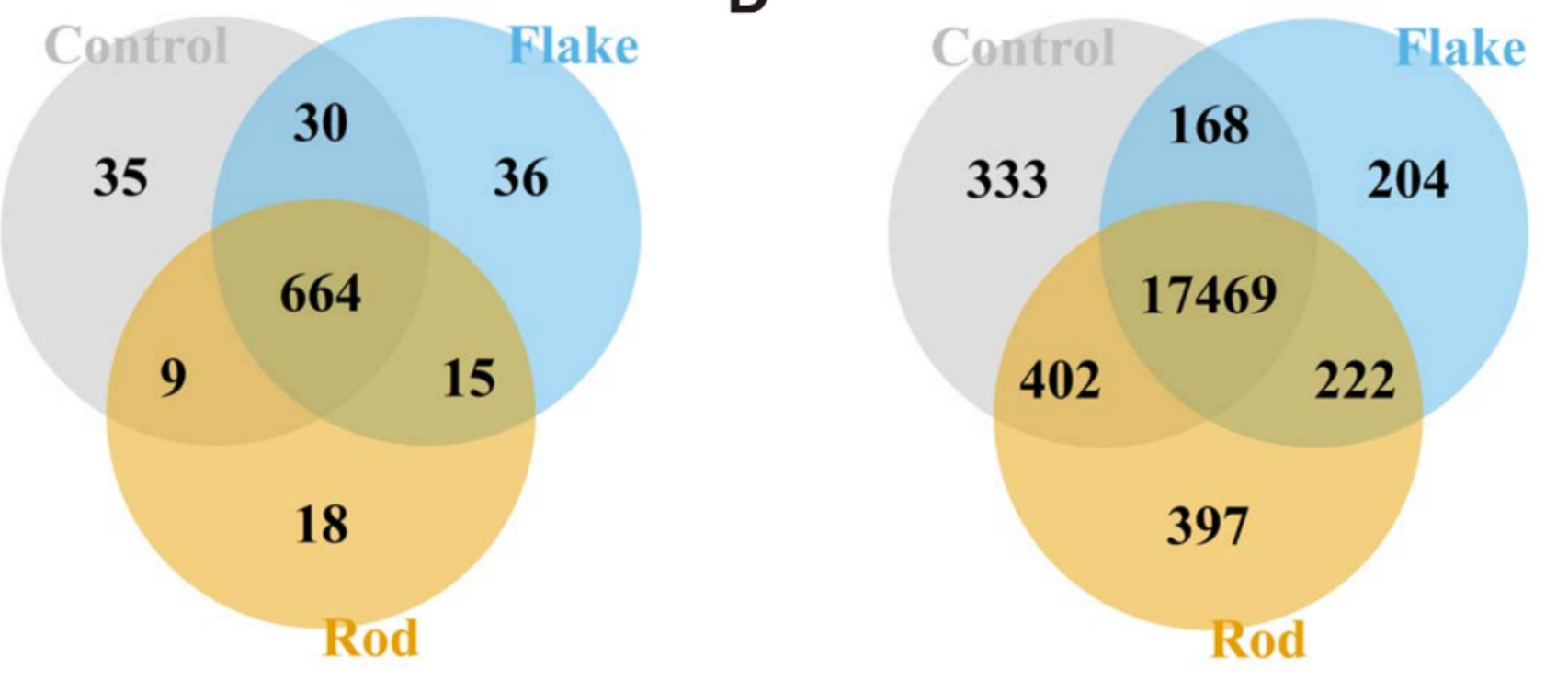

Figure 1

Scoring plots for principal components analysis (PCA) and Venn diagram of small RNA (miRNAs) and transcriptome (mRNAs) sequencing in rat ASTs exposed to two different shapes (flake or rod) of $y$-Al203NPs at $125 \mu \mathrm{g} / \mathrm{mL}$ for $72 \mathrm{~h}(\mathrm{n}=3)$. PCA plots of the expression profiles of miRNAs $(A)$ and mRNAs (B). Each sample is represented with a single point, with different colors and shapes for each group (Control, Flake, and Rod). Venn diagrams show the numbers of the significantly changed expression profiles of miRNAs (C) and mRNAs (D) among Control, Flake, and Rod groups. 

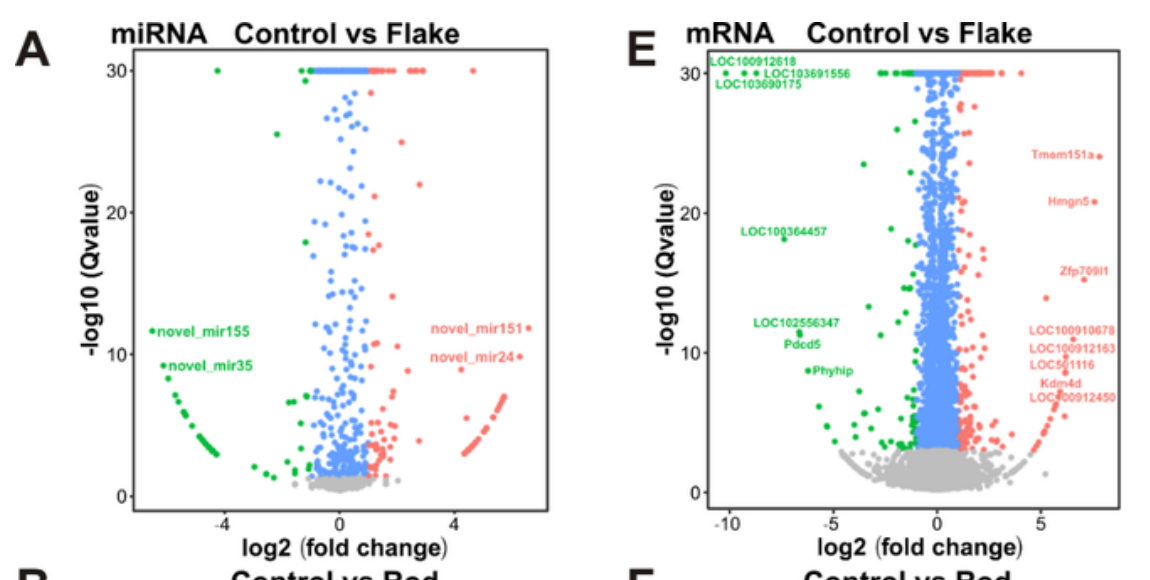

B
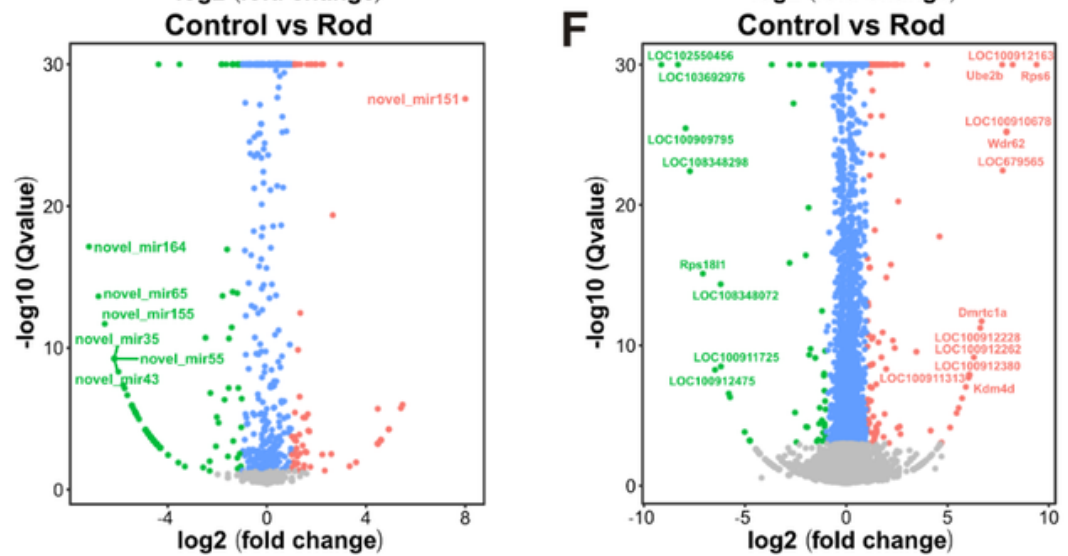

C

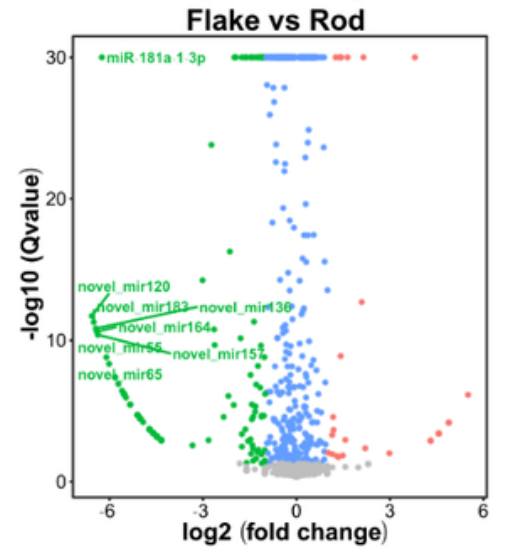

D
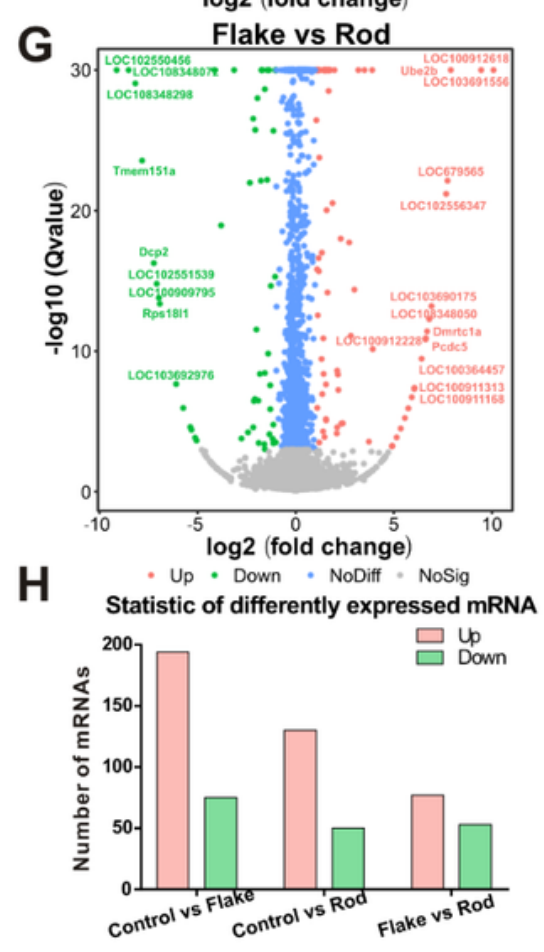

\section{Figure 2}

Volcano plots of miRNAs (A-C) and mRNAs (E-G) in rat ASTs exposed to two different shapes (flake or rod) of $\gamma$-Al203-NPs at $125 \mu \mathrm{g} / \mathrm{mL}$ for $72 \mathrm{~h}(\mathrm{n}=3)$. Each point represents a miRNA or mRNA, The expression level and negative logarithm of Q value (FDR) as the abscissa and ordinate, respectively. Pink and green dots represent the significantly up-regulated and down-regulated genes, respectively. Blue or gray dots represent miRNAs or mRNAs that were not differentially expressed. The total number of 
significantly differentially expressed genes are shown for miRNAs $(D)$ and mRNAs $(H)$. Group comparisons: Control vs Flake (A and E); Control vs Rod (B and F); Flake vs Rod (C and G).
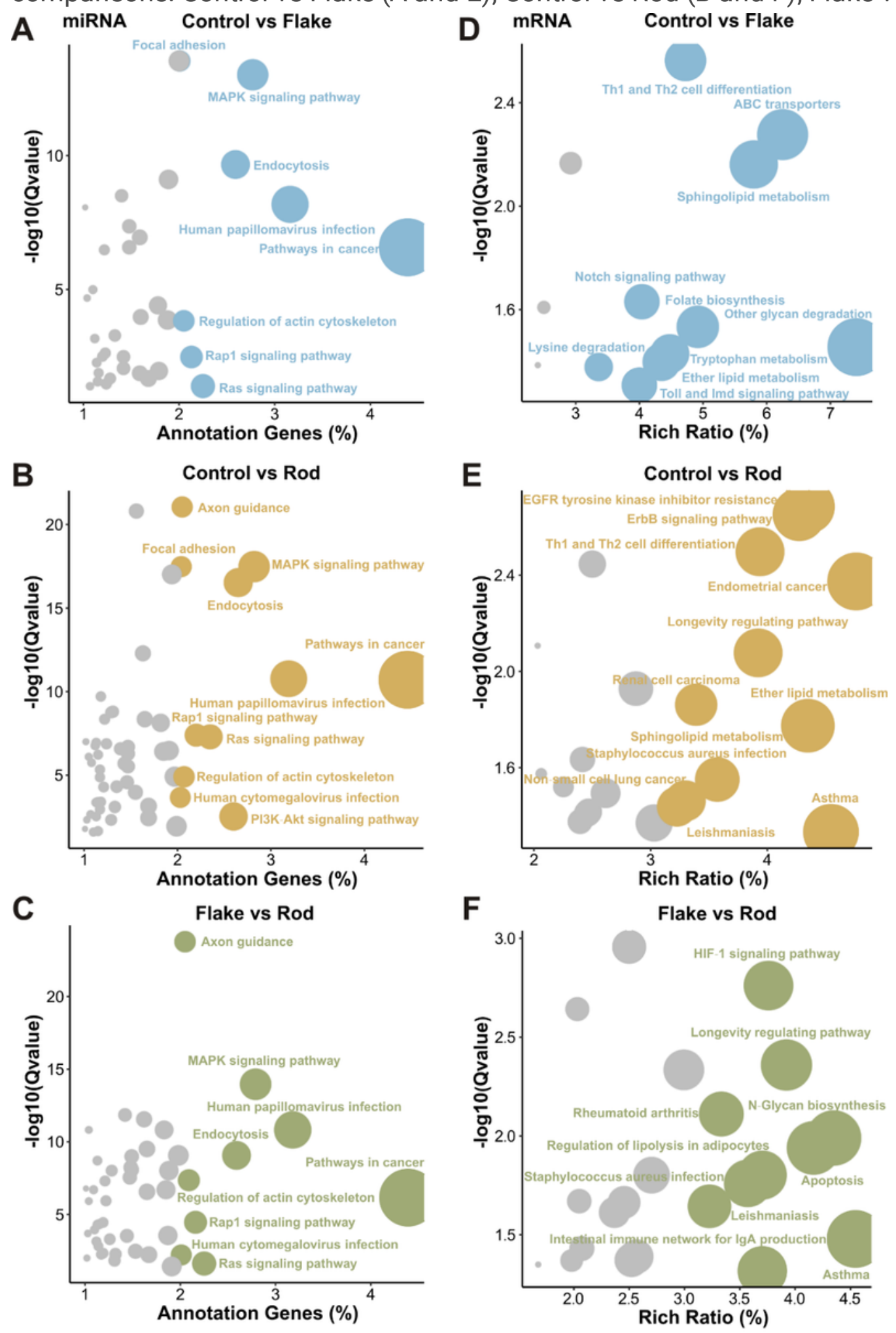

Figure 3

Bubble plots reveal the KEGG pathway enrichment analysis of significantly differentially expressed miRNAs (A-C) and mRNAs (D-F) in rat ASTs exposed to two different shapes (flake or rod) of $\mathrm{Y}$-Al203-NPs at $125 \mu \mathrm{g} / \mathrm{mL}$ for $72 \mathrm{~h}(\mathrm{n}=3)$. The vertical axis indicates the negative $\log (10)$ of $\mathrm{Q}$ value (FDR) of pathway 
enrichment analysis, and horizontal axis represents the number of genes annotated to the pathway, accounting for the proportion of the total number of genes. The size of the circle indicated the number of enriched miRNAs or genes. Group comparisons: Control vs Flake (A and D); Control vs Rod (B and E); Flake vs Rod ( $\mathrm{C}$ and $\mathrm{F}$ ).

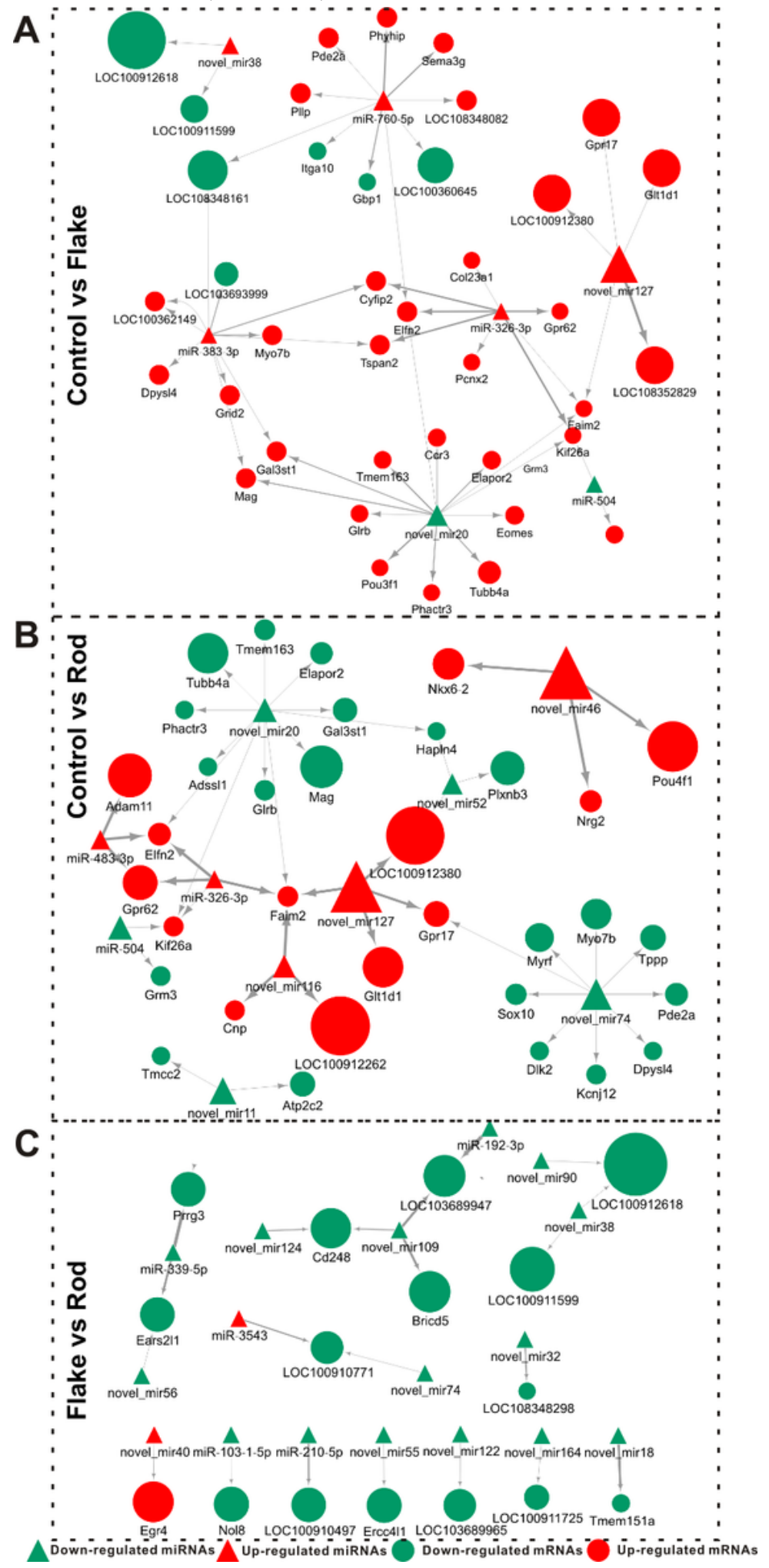

Figure 4 
MiRNA and gene interaction network analysis for the significantly differentially expressed genes in rat ASTs exposed to two different shapes (flake or rod) of $\mathrm{Y}$-Al203-NPs at $125 \mu \mathrm{g} / \mathrm{mL}$ for $72 \mathrm{~h}(\mathrm{n}=3$ ). (A) Control vs Flake; (B) Control vs Rod; (C) Flake vs Rod. The triangle and circle (nodes) represent the significantly differentially expressed miRNAs and genes, respectively. The thickness of the connecting line (edges) between miRNA and gene reflects the strength of their interactions. The node size represents the abundance of the expressions of miRNAs and genes. Red and green colors represent the positive and negative correlations, respectively.
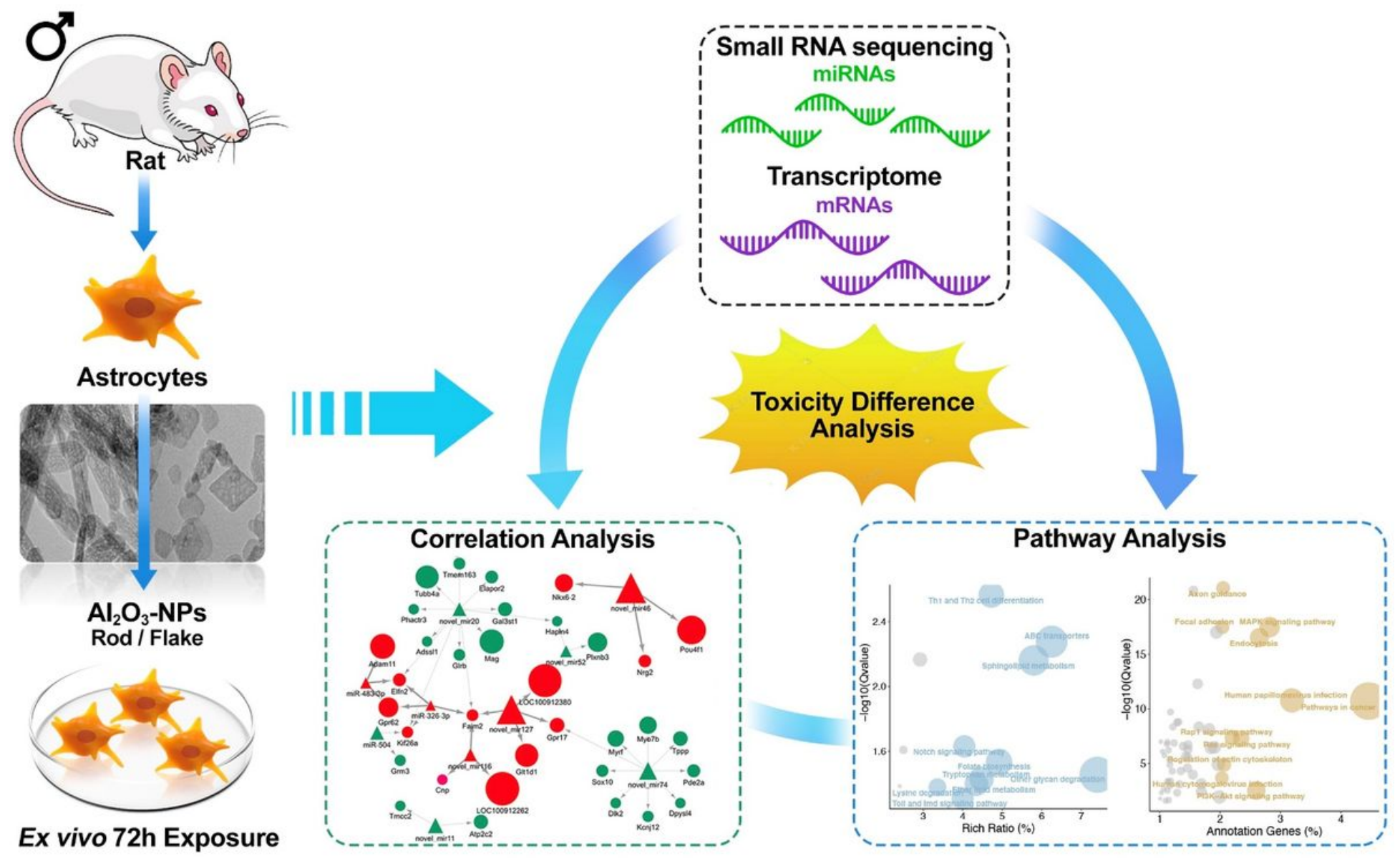

\section{Ex vivo 72h Exposure}

\section{Figure 5}

The graph abstrate of this study was to elucidate the toxicity of two different shapes of $\mathrm{Y}$-Al2O3-NPs (flake or rod) to primary rat astrocytes at a dose of $125 \mu \mathrm{g} / \mathrm{mL}$ for $72 \mathrm{~h}$ by small RNA sequencing and transcriptome analysis.

\section{Supplementary Files}

This is a list of supplementary files associated with this preprint. Click to download.

- SI20210607CYY.docx 